Cored Bandrés, S., Vázquez Toledo, S., Liesa Orús, M. y Baldassarri, S. (2021). La potencialidad de la tecnología en la medición del desarrollo de habilidades sociales en niños con TEA: un análisis desde parámetros fisiológicos. Revista de Investigación Educativa, 39(2), 445-462.

DOI: http://dx.doi.org/10.6018/rie.430891

\title{
La potencialidad de la tecnología en la medición del desarrollo de habilidades sociales en niños con TEA: un análisis desde parámetros fisiológicos
}

\author{
The potential of technology in measuring the development of \\ social skills in children with ASD: an analysis from physiological \\ parameters
}

\author{
Sergio Cored Bandrés*, Sandra Vázquez Toledo**, Marta Liesa Orús* y Sandra Baldassarri*** \\ *Departamento de Ciencias de la Educación. Facultad de Ciencias Humanas y de la Educación. \\ Universidad de Zaragoza (España) \\ **Departamento de Ciencias de la Educación. Facultad de Educación. Universidad de Zaragoza (España) \\ ***Departamento de Informática e Ingeniería de Sistemas. Escuela de Ingeniería y Arquitectura.
}

Universidad de Zaragoza (España)

\begin{abstract}
Resumen
El uso pedagógico de la tecnología es una realidad y está impulsando un cambio importante en la atención educativa a los niños con necesidades educativas especiales. Sin embargo, pocos son los estudios centrados en cómo verificar el impacto de estas herramientas en las diversas intervenciones que se realizan. En este sentido y con el objeto de evaluar mediante wearables los cambios reales que se producen cuando se interviene en el ámbito de las habilidades sociales en niños con TEA y verificar la potencialidad didáctica de los dispositivos tecnológicos, se plantea una investigación de diseño mixto basada en el método estudio de casos. La muestra ha estado
\end{abstract}

\footnotetext{
Los autores queremos agradecer el apoyo económico y logístico del contrato RTI2018-096986-B-C31 del Ministerio de Ciencia, Innovación y Universidades de España, del Departamento de Ciencia, Universidad y Sociedad del Conocimiento del Gobierno de Aragón (Contrato de personal investigador predoctoral en formación) y de los grupos de investigación Educación y Diversidad (EDI) S49_17R y AffectiveLab-T60-20R. Correspondencia: Sergio Cored Bandrés, scoban@unizar.es, Facultad de Ciencias Humanas y de la Educación, C/ Valentín Carderera, 4, 22003, Huesca.
} 
constituida por cuatro alumnos con TEA. La recolección de datos se ha llevado a cabo, por un lado, mediante la observación sistemática de las conductas durante las sesiones de intervención, y por otro, mediante la medición de parámetros fisiológicos (ritmo cardíaco y actividad electrodérmica) a través de un wearable -Empatica E4-, asociándose a estados emocionales de los niños. Los resultados reflejan un aumento del ritmo cardíaco y variaciones en la actividad electrodérmica en situaciones de incomprensión y espera de turnos y un estado más calmado (disminución del ritmo cardíaco) en momentos de fijación de la atención en el dispositivo tecnológico. Asimismo, se observa un descenso en comportamientos no sociales como estereotipias o la mirada perdida y un aumento en conductas sociales. En concreto, aparecen incrementos significativos en la atención conjunta y en el contacto ocular.

Palabras clave: Trastorno del Espectro Autista (TEA); necesidades educativas especiales; tecnologías de la Información y de la Comunicación (TIC); wearables; parámetros fisiológicos; habilidades sociales

\section{Abstract}

The pedagogical use of technology is a reality and it is encouraging a major change in educational care for children with special educational needs. However, few studies have focused on how to verify the impact of these tools on the interventions that are carried out. In this sense, and in order to evaluate through wearables the actual changes that take place when intervening in the field of social skills in children with ASD and verify the didactic potential of the technological devices, a mixed-design research based on the case study method is proposed. The sample is made up of four students with ASD. Data collection has been carried out, on the one hand, through systematic observation of behaviours during the intervention sessions, and on the other hand, by measuring physiological parameters (heart rate and electrodermal activity) through a wearable -Empatica E4-. Those factors have then been associated with the emotional states of children. The results show an increase in heart rate and variations in electrodermal activity in situations of misunderstanding and waiting for turns. A calmer state (decreased heart rate) is observed in moments of fixation of attention on the technological device. Likewise, there is a decrease in non-social behaviours such as stereotypies or lost gaze and an increase in social behaviours. Specifically, there are significant increases in joint attention and eye contact.

Keywords: Autism Spectrum Disorder (ASD); special educational needs; Information and Communication Technology (ICT); wearables; physiological parameters; social skills

\section{Introducción}

Las ideas asociadas al Trastorno del Espectro del Autista (en adelante, TEA) se han ido modificando a lo largo del tiempo. En la actualidad, la Asociación Americana de Psiquiatría (2014), enmarca al TEA en los trastornos del desarrollo neurológico, describiendo dos ámbitos principalmente afectados, la comunicación e interacción social y la aparición de patrones repetitivos y restringidos de conducta.

Uno de los aspectos en los que mayores dificultades presentan estos niños, y que aparecen dentro del ámbito de comunicación e interacción social, son las habilidades sociales (en adelante hh.ss.), definidas por Monjas (2009) como un "conjunto de cogni- 
ciones, emociones y conductas que permiten relacionarse y convivir con otras personas de forma satisfactoria y eficaz" (p. 39). Éstas incluyen tanto comunicación verbal como no verbal. Actualmente se considera que abarcan 3 dimensiones (Ibidem), estando las dos últimas en estrecha relación con la Teoría de la Mente:

- Las conductas observables como la mirada, expresión facial, gestos, forma, contenido de la comunicación verbal, etc.

- Los componentes cognitivos que hacen referencia a nuestros pensamientos y creencias -nuestra forma de percibir y evaluar la realidad-.

- Los componentes emocionales que nos permiten comprender nuestras emociones y las de los demás y regularlas para facilitar las relaciones.

Como se puede apreciar, las hh.ss. resultan imprescindibles para la interacción e inclusión social, para la relación con el entorno. Por ello, se convierten en áreas de especial interés en la intervención educativa (De la Iglesia y Olivar, 2008), constituyéndose como objetivos a alcanzar en los programas educativos. Diversos autores defienden la idea de que las hh.ss. son conductas aprendidas (Kelly, 2000; Liesa, 2002). Considerando esta percepción, las personas con dificultades en este ámbito podrán mejorar sus hh.ss. llevando a cabo un entrenamiento sistemático de las mismas. Diferentes investigaciones (Mendo, 2019; Peñaherrera-Vélez et al., 2019) demuestran que el desarrollo de programas específicos de intervención en hh.ss. permiten su mejora e incremento. Asimismo, otros estudios han constatado que este tipo de programas también son efectivos en personas con TEA (Bermúdez et al., 2020; Peters y Thompson, 2015).

Teniendo en cuenta el progreso de la sociedad, es comprensible y necesario que los profesionales de la educación incorporen nuevas metodologías y herramientas para cubrir las necesidades de este alumnado de una manera mucho más eficaz. Una de esas herramientas es la tecnología, que "ha transformado el escenario social incorporando estrategias, técnicas y métodos para obtener procesos educativos más significativos" (Caballero-González y García-Valcárcel, 2020, p.1).

En concreto, en personas con TEA, son múltiples las ventajas que nos aporta su implementación. Diversas investigaciones (Hardy et al., 2002; Neale et al., 2002) han señalado que la tecnología ofrece a este alumnado un entorno controlado, pues ayudan a estructurar y organizar el entorno de interacción al configurarse como un medio muy predictible que ofrece contingencias comprensibles para el alumno. Asimismo, ofrece la posibilidad de adaptar el ritmo de aprendizaje a las características del alumnado, trabajar de una manera multidisciplinar $\mathrm{u}$ ofrecer al propio individuo la oportunidad de experimentar e interactuar de forma sencilla e intuitiva con la información que se le muestra, sin la presencia de estímulos distractores (Boyd et al., 2015; Crescenzi-Lanna y Grané-Oró, 2016). Además, facilitan la creación de un entorno dinámico, atractivo y motivador para los alumnos con TEA, por su ritmo y combinación de gráficos, sonidos y animaciones, favoreciendo la recepción de estímulos de manera multisensorial, preferiblemente visual (Parsons et al., 2016). Por otro lado, numerosas investigaciones (Jiménez et al., 2017; Vahabzadeh et al., 2018) han demostrado que la tecnología tiene un impacto en el desarrollo de competencias emocionales y sociales y en la propia autorregulación, mejorando los procesos de interacción y comunicación. 
Pero las TIC no solo permiten compensar y apoyar las diversas intervenciones que se llevan a cabo con los niños, sino también, evaluar de una manera eficiente y objetiva el impacto de las mismas en el desarrollo y adquisición de las hh.ss. Este hecho resulta muy complejo en este alumnado debido a las dificultades de expresión y reconocimiento de las emociones que presentan (Jodra y García-Villamisar, 2019).

El uso de wearables permite detectar diferentes parámetros fisiológicos como el ritmo cardíaco (en adelante HR) o la actividad electrodérmica (en adelante EDA), que posibilitan discernir estados afectivos (Krupa et al, 2016; Sarabadani et al. 2018). La EDA se define como "la variación en las propiedades de la piel como resultado de la transpiración" (Pijeira-Diaz et al., 2018, p. 400) y, junto con el HR, han sido ampliamente utilizados debido a su relación directa con las respuestas emocionales, permitiendo captar los momentos de excitación o estrés (Boucsein, 2012). Sin embargo, aunque diversos estudios han investigado los niveles de estrés en adultos (Sano y Picard, 2013), los wearables han sido utilizados en menor medida como herramienta para medir excitación emocional en niños con dificultades en comunicación (Aparicio et al., 2018). No obstante, es importante remarcar que este tipo de dispositivos han demostrado ser de gran utilidad para personas con TEA, para detectar emociones (Sarabadani et al., 2018), mejorar su calidad de vida (Koo et al, 2018) o en la evaluación del comportamiento social (Kowallik y Schweinberger, 2019).

Considerando los antecedentes teóricos expuestos y en aras de contribuir al respaldo de evidencias que dan soporte a la eficacia de la tecnología en el desarrollo de hh.ss. en niños TEA y al uso de wearables para medir el impacto de las intervenciones, se ha diseñado e implementado un programa de intervención en un colegio público de escolarización preferente TEA de Aragón. Este programa se ha fundamentado en el Programa de Asertividad y Habilidades Sociales (PAHS), diseñado por Monjas en 2009 y en el Manual de Teoría de la Mente para niños con autismo (Cornago et al., 2012).

En él han participado cuatro alumnos con los que se ha trabajado agrupándolos por parejas. Las sesiones, de una hora de duración, han tenido una periodicidad semanal durante cuatro meses. Con los sujetos 1 y 2 se han realizado 10 sesiones y con los sujetos 3 y 4, un total de 11. En ellas y, mediante el uso de la tecnología (en concreto un Ipad), se han trabajado contenidos sobre la teoría de la Mente, definida como "habilidad cognitiva compleja, que permite que un individuo atribuya estados mentales a sí mismo y a otros" (Uribe et al., 2010, p.1) y elementos de la teoría de coherencia central débil que nos habla sobre la problemática en la capacidad de integrar información (López y Leekan, 2007). Ambos aspectos están íntimamente relacionados con las hh.ss., y aparecen afectados en las personas con TEA (Jodra, 2015). Durante la intervención, se ha utilizado la pulsera Empatica E4 como herramienta de evaluación y de recogida de datos fisiológicos en 3 de los 4 sujetos.

\section{Método}

Este trabajo de investigación se ha fundamentado en un diseño mixto, basado en el concepto de triangulación de métodos para recabar información mediante técnicas cuantitativas y cualitativas, facilitando así un mayor entendimiento del fenómeno 
estudiado (Rodríguez, 2012). Asimismo, el método utilizado ha sido el estudio de casos, el cual se define como "una investigación empírica que estudia un fenómeno contemporáneo en su entorno de vida real, específicamente cuando los límites entre el fenómeno y su contexto no son claramente evidentes" (Yin, 2008, p.13). Álvarez y San Fabián (2012) añaden que este método se distingue por ser particularista, sin olvidar el contexto, descriptivo y holístico.

\section{Objetivos}

El objetivo principal que se persigue en esta investigación es evaluar mediante wearables el impacto de una intervención educativa a través de parámetros fisiológicos (ritmo cardíaco y actividad electrodérmica), asociando los mismos a situaciones concretas y analizando el estrés y excitación generada.

Por otro lado, este estudio también se plantea analizar y comprender la potencialidad educativa de la aplicabilidad de tecnología en la mejora de las hh.ss. en alumnos con TEA. Concretamente, se pretende evaluar el impacto de un programa de hh.ss. en un grupo de niños que presenta este cuadro mediante el uso de la tecnología.

\section{Población y Muestra}

Para la presente investigación se llevó a cabo un procedimiento de muestreo no probabilístico, concretamente se ha utilizado el muestreo intencional o de conveniencia. Por ello, los participantes del estudio fueron seleccionados de un centro escolar público de atención preferente TEA. Como criterios de inclusión se tuvieron en cuenta que los individuos hubieran sido diagnosticados con TEA y presentaran lenguaje oral, obteniendo finalmente una muestra de 4 sujetos (Tabla 1).

Tabla 1

Características de los sujetos de la muestra

\begin{tabular}{|c|c|c|c|c|}
\hline Sujetos & Edad & $\begin{array}{l}\text { Curso escolar } \\
\text { (Desfase } \\
\text { curricular) }\end{array}$ & $\begin{array}{c}\text { Nivel de } \\
\text { gravedad } \\
\text { del TEA }\end{array}$ & $\begin{array}{l}\text { Desarrollo comunicación e } \\
\text { interacción social }\end{array}$ \\
\hline Sujeto 1 & 6 años & $\begin{array}{l}1^{\circ} \text { de primaria } \\
\quad(1 \text { año })\end{array}$ & Grado 1 & $\begin{array}{l}\text { Lenguaje muy funcional. } \\
\text { Presenta alteraciones en la } \\
\text { comunicación (uso de ecolalias) } \\
\text { y dificultad para relacionarse } \\
\text { con sus iguales. }\end{array}$ \\
\hline Sujeto 2 & 6 años & $\begin{array}{l}1^{\circ} \text { de primaria } \\
\qquad(2 \text { años })\end{array}$ & Grado 2 & $\begin{array}{l}\text { Lenguaje poco funcional. } \\
\text { Presenta grandes dificultades } \\
\text { en atención conjunta y relación } \\
\text { con otros. Alto número de } \\
\text { ecolalias diferidas sin función } \\
\text { comunicativa. }\end{array}$ \\
\hline
\end{tabular}




\begin{tabular}{|c|c|c|c|c|}
\hline Sujetos & Edad & $\begin{array}{l}\text { Curso escolar } \\
\text { (Desfase } \\
\text { curricular) }\end{array}$ & $\begin{array}{c}\text { Nivel de } \\
\text { gravedad } \\
\text { del TEA }\end{array}$ & $\begin{array}{l}\text { Desarrollo comunicación e } \\
\text { interacción social }\end{array}$ \\
\hline Sujeto 3 & 11 años & $\begin{array}{l}4^{\circ} \text { de primaria } \\
\quad(2 \text { años })\end{array}$ & Grado 1 & $\begin{array}{l}\text { Lenguaje muy funcional. } \\
\text { Manifiesta dificultades en } \\
\text { ámbito pragmático. Dificultades } \\
\text { para controlar sus impulsos } \\
\text { lo que supone un problema a } \\
\text { la hora de interactuar con los } \\
\text { demás. }\end{array}$ \\
\hline Sujeto 4 & 10 años & $\begin{array}{l}3^{\circ} \text { de primaria } \\
\quad(1 \text { año })\end{array}$ & Grado 1 & $\begin{array}{l}\text { Lenguaje funcional. Presenta } \\
\text { dificultades significativas } \\
\text { en ámbito pragmático, } \\
\text { resultándole complejas las } \\
\text { interacciones sociales con otros }\end{array}$ \\
\hline
\end{tabular}

\section{Instrumento}

Como procedimiento esencial de recogida de datos se utilizó la observación participante. Se grabaron la totalidad de las sesiones para garantizar la máxima precisión en la recogida de los mismos.

Para la sistematización de los datos se utilizó el cuestionario de Arias-Pujol et al. (2015) durante los visionados de las sesiones. Este cuestionario permite registrar conductas en relación a dos grandes categorías: conductas de no interacción social recíproca (no_ISR) compuesta por 12 ítems y conductas de interacción social recíproca conformada por 22 ítems. Dentro de esta última decidimos llevar a cabo una distinción entre interacción social con el investigador (ISR) e interacción social con el compañero (ISRC). Asimismo, y de manera complementaria se utilizó el protocolo de observación de la Interacción del Alumnado (PIA) (Pedrosa et al., 2013). En concreto, la categoría 2 que hace referencia a la interacción negativa y la categoría 5 que incluye aquellos comportamientos inobservables o no clasificables (INC).

Por otro lado, se utilizó la pulsera Empatica E4, un wearable multisensor no intrusivo que permite medir parámetros como temperatura, ritmo cardíaco, actividad electrodérmica o presión arterial (Garbarino et al., 2014). Este dispositivo cuenta con una aplicación de escritorio para transferir datos a un repositorio en la nube, una aplicación web, para ver y gestionar la información y una aplicación móvil para transmitir, ver y procesar los datos en tiempo real.

\section{Procedimiento de recogida y análisis de datos}

Para codificar y registrar las conductas de las sesiones grabadas se contó con el software observacional LINCE PLUS (Soto et al., 2019). Este programa permite la detección de patrones conductuales y su categorización de manera sencilla e intuitiva, facilitando la tarea al observador encargado de visionar las grabaciones. 
Asimismo, los datos obtenidos a través del software se analizaron mediante el programa estadístico SPSS versión 26, calculando las frecuencias relativas expresadas en tanto por ciento de las conductas observadas en cada una de las 21 sesiones de las que consta la intervención.

Por otra parte, se analizó la información extraída de la pulsera Empatica E4. En primer lugar, se identificaron los picos observados para, posteriormente, triangular esos datos con las imágenes grabadas y determinar en qué momentos concretos se observan alteraciones en los parámetros fisiológicos asociados al estrés y la excitación.

En este sentido, la triangulación ha permitido contraponer los datos, complementar los resultados y verificar tendencias para tener una visión más aproximada de la realidad. Así como garantizar la validez, credibilidad y dotar de rigor a los resultados.

\section{Resultados}

\section{Registro de las conductas}

Para facilitar la comprensión y la descripción de los resultados se ha decidido plasmar los datos obtenidos en cuatro de las sesiones. Entre ellas se encuentran la primera y la última para conocer el impacto real de la intervención sobre las conductas observadas y, por otro lado, dos sesiones intermedias que permiten registrar cómo ha sido la variación de esas conductas durante la intervención.

En relación al sujeto 1, los resultados que se presentan a continuación corresponden a las sesiones 1, 4, 7 y 10 (Tabla 2).

Tabla 2

Frecuencias relativas categorías generales sujeto 1

\begin{tabular}{ccccc}
\hline No Sesión & No_ISR & ISR & ISRC & INC \\
\hline 1 & $30,6 \%$ & $67,4 \%$ & $1,4 \%$ & $0,6 \%$ \\
4 & $22,7 \%$ & $62,5 \%$ & $14,8 \%$ & $0 \%$ \\
7 & $12,5 \%$ & $59,6 \%$ & $27,9 \%$ & $0 \%$ \\
10 & $11,2 \%$ & $62,2 \%$ & $26,6 \%$ & $0 \%$ \\
\hline
\end{tabular}

En primer lugar, teniendo en cuenta los resultados obtenidos a nivel global en las 4 categorías de conductas observadas, se aprecia una disminución significativa en la categoría no_ISR a lo largo de las diferentes sesiones. En cambio, la categoría ISRC aumenta de manera muy notable, creciendo del 1,4\% al 26,5\% del total de las conductas observadas. Tanto en ISR como en INC no aparecen variaciones relevantes.

Profundizando en las conductas no_ISR, se observa un descenso en las estereotipias tanto verbales como motoras del $19,4 \%$ al $2 \%$, así como en la conducta de mirada perdida (MP), que se reduce del $1,4 \%$ al $0 \%$. 
Con respecto a la categoría ISR, y aunque de manera global no presente cambios significativos, cabe destacar el incremento en la atención conjunta, del $20,1 \%$ en la primera sesión al 37,7\% en la última. En concreto, se percibe un aumento en dos de las conductas que forman parte de la atención conjunta: en las aproximaciones a frases (AFP) en más de 6 puntos porcentuales hasta alcanzar un $8,2 \%$ y en las frases $(\mathrm{F})$ que crecen del 1,4\% al 15,3\%. Por último, en relación a las conductas ISRC, la atención conjunta aumenta de manera muy significativa hasta el 17,3\% y el contacto corporal, suma de los contactos breves (CCBC) y mantenidos (CCMC), también se incrementa considerablemente del $0 \%$ hasta el 6,1\%.

En cuanto al sujeto 2, los resultados plasmados hacen referencia a las sesiones 1 , 4, 8 y 10 (Tabla 3).

Tabla 3

Frecuencias relativas categorías generales sujeto 2

\begin{tabular}{ccccc}
\hline No Sesión & No_ISR & ISR & ISRC & INC \\
\hline 1 & $60,9 \%$ & $37,2 \%$ & $1,3 \%$ & $0,6 \%$ \\
4 & $51,1 \%$ & $46,7 \%$ & $2,2 \%$ & $0 \%$ \\
8 & $34,8 \%$ & $58,1 \%$ & $7,1 \%$ & $0 \%$ \\
10 & $20,2 \%$ & $59,6 \%$ & $20,2 \%$ & $0 \%$ \\
\hline
\end{tabular}

Teniendo en cuenta las 4 grandes categorías, se aprecia una disminución significativa de las conductas no_ISR y un aumento tanto en ISR como en ISRC.

Si se ahonda en cada una de ellas, en relación a las conductas no_ISR se percibe un descenso en estereotipias, tanto verbales como motoras. En la sesión 1 representan el 20,5\% y en la sesión 10 tan solo el 1,1\%. Asimismo, la mirada perdida también disminuye más de 10 puntos porcentuales hasta situarse en el 3,4\%. Por último, la conducta de abandono del lugar de trabajo (AT) que representa el 5,8 \% del total de las conductas en la primera sesión, desaparece en las últimas.

Por otro lado, la atención conjunta con el investigador aumenta significativamente del $7,7 \%$ al $28 \%$, destacando dentro de este tipo de conductas el uso de la aproximación a frase (AFP), la cual crece del 0 al 2,2\%. Además, el contacto ocular con el investigador pasa de un 4,5\% al comienzo de la intervención y alcanza el 14,6\% en la última sesión.

Finalmente, en lo referente al ISRC, aumenta la atención conjunta del $0,6 \%$ al $14,5 \%$, siendo las emisiones de palabras (PAC) las que más se incrementan dentro de la misma. De igual manera y como sucede con la ISR, el contacto ocular con el otro alumno también registra un incremento significativo del $0 \%$ al $4,5 \%$.

En lo relativo al sujeto 3, los resultados reflejados corresponden a las sesiones 1, $4,8$ y 11 (Tabla 4$)$. 
Tabla 4

Frecuencias relativas categorías generales sujeto 3

\begin{tabular}{ccccc}
\hline No Sesión & No_ISR & ISR & ISRC & INC \\
\hline 1 & $18,5 \%$ & $62,6 \%$ & $18,9 \%$ & $0 \%$ \\
4 & $11 \%$ & $63,8 \%$ & $25,2 \%$ & $0 \%$ \\
8 & $5 \%$ & $70 \%$ & $25 \%$ & $0 \%$ \\
11 & $3 \%$ & $79,1 \%$ & $17,9 \%$ & $0 \%$ \\
\hline
\end{tabular}

En relación a la categoría no_ISR hay una reducción muy significativa de las conductas que la conforman. Por otro lado, las conductas ISR aumentan sustancialmente. Tanto las interacciones con el compañero como las conductas INC, no presentan cambios reseñables.

Adentrándose en los resultados de la categoría no_ISR destaca el gran descenso en los comportamientos inadecuados (CI), del 7\% al 0,7\%.

Por otra parte, en las conductas asociadas al ISR destaca el crecimiento de aquellas relacionadas con la atención conjunta, pasando del 21,5 al 41,1\% y, en particular, del uso de palabras, cuyo incremento es del $10 \%$.

Respecto a la ISRC, aunque de manera global no presente variaciones relevantes hay dos conductas que sí aumentan de forma significativa. Por un lado, la atención conjunta que se duplica, situándose en el 12\%. Por otro, el contacto ocular (COC) que aumenta del 1,3\% al 3,7\%.

Para el sujeto 4, se han tenido en cuenta los resultados de las sesiones 1, 4, 7 y 11 (Tabla 5).

Tabla 5

Frecuencias relativas categorías generales sujeto 4

\begin{tabular}{ccccc}
\hline No Sesión & No_ISR & ISR & ISRC & INC \\
\hline 1 & $22,4 \%$ & $55,2 \%$ & $22,4 \%$ & $0 \%$ \\
4 & $16,4 \%$ & $56,8 \%$ & $24,7 \%$ & $2,1 \%$ \\
7 & $16 \%$ & $62,8 \%$ & $19,1 \%$ & $2,1 \%$ \\
11 & $12,2 \%$ & $65,4 \%$ & $22,4 \%$ & $0 \%$ \\
\hline
\end{tabular}

En cuanto a las 4 categorías, hay una reducción a la mitad de las conductas asociadas a no_ISR y un aumento significativo de las conductas ISR. No aparecen variaciones relevantes ni en ISRC ni en las conductas INC.

Dentro de las conductas vinculadas a la categoría no_ISR aparece un importante descenso en la Mirada Perdida (MP) del 9,7\% al 3,1\%. Asimismo, los comportamientos 
inadecuados (CI), que en la sesión 1 conformaban el 1,8\% del total, desaparecen en las últimas sesiones.

Con respecto a las conductas asociadas a la categoría ISR, son dos las que manifiestan un incremento considerable. Por un lado, la atención conjunta alcanza el 44,1\%, creciendo casi 20 puntos desde la primera sesión. En concreto, aumentan de manera muy significativa las aproximaciones a frases (se cuadruplican) y las frases que se multiplican por dos. Del mismo modo el contacto ocular con el investigador crece del $6,1 \%$ al $16,7 \%$.

Por último, aunque el ISRC se mantiene constante, la atención conjunta con el compañero se incrementa $8,8 \%$ hasta alcanzar el $14,2 \%$, destacando dentro de la misma el aumento de las palabras (PAC) en más de un $6 \%$.

\section{Detección de los parámetros fisiológicos}

A continuación, se presentan los datos fisiológicos obtenidos de los 3 sujetos que utilizaron la Empatica. Como punto de partida, es importante tener en cuenta que tanto el aumento de HR como la variación de EDA se relacionan con la excitación o aumento del estrés, mientras que la disminución del HR se asocia a estados de calma.

En relación al sujeto 1 se observan aumentos en el HR cuando muestra dificultades en una tarea o si ésta le motiva -le despierta curiosidad o interés-. Asimismo, estos cambios también aparecen en momentos de espera de turno, sobre todo si el compañero falla o tarda en realizar el ejercicio, y cuando comienza una tarea, pero el sujeto no es quien la inicia. En cambio, el descenso de HR aparece cuando fija su atención en el Ipad y no está pendiente de su compañero, en el tiempo de transición entre las tareas, centrando la atención la mayoría de las ocasiones en el Ipad, o cuando comprende el ejercicio que se le propone.

En lo relativo a la EDA, se perciben alteraciones significativas cuando el sujeto no entiende la actividad, cuando la tarea va a finalizar y en los momentos en los que el alumno se levanta y abandona el lugar de trabajo.

Si ahondamos en los datos fisiológicos extraídos del sujeto 3, se observa un aumento en HR en situaciones en las que el sujeto no entiende un ejercicio que se está realizando o si la tarea le gusta, es motivante para él y la resuelve de manera correcta. Asimismo, estas variaciones también aparecen cuando el alumno se cansa de esperar su turno o quiere empezar él -a pesar de ser el turno de otro compañero-. También sucede esto cuando el otro alumno se equivoca o tarda más de lo esperado en resolver algún ejercicio y en el momento final de la sesión en el que pueden jugar con la aplicación que ellos elijan. Cabe destacar que este aumento en HR, también aparece segundos después de que el sujeto agreda al compañero.

Por otro lado, los grandes descensos de HR se han asociado a momentos en los que el alumno fija su atención en el Ipad mientras espera su turno o en las transiciones entre actividad y actividad en los que, de la misma manera, centra la atención en el Ipad.

Relacionado con la EDA, aparecen variaciones significativas vinculadas a situaciones en las que el sujeto no entiende la actividad propuesta, cuando una actividad concreta 
está terminando o en momentos en los que el alumno se salta los turnos porque no es capaz de esperar.

Asimismo, profundizando en los resultados obtenidos en los parámetros fisiológicos del sujeto 4, aparecen picos positivos en el HR cuando el sujeto muestra dificultades en la tarea o en momentos en que el ejercicio propuesto le motiva y le gusta. En cambio, los descensos significativos registrados se asocian a situaciones en las que el alumno entiende la tarea y la lleva a cabo sin dificultades o cuando el sujeto mira atentamente el Ipad mientras espera que llegue su turno.

Por otra parte, aparecen alteraciones en la EDA en momentos en los que el investigador explica un ejercicio o cuando la actividad propuesta implica tareas de gran complejidad.

A modo de síntesis, en la tabla 6 se presenta una comparativa de los datos obtenidos de los diferentes sujetos.

Tabla 6

Relación entre los cambios significativos en datos fisiológicos obtenidos y las situaciones concretas de las sesiones

\begin{tabular}{|c|c|c|c|c|c|c|c|c|c|}
\hline \multirow{2}{*}{$\begin{array}{c}\text { Situaciones/ } \\
\text { Conductas }\end{array}$} & \multicolumn{3}{|c|}{ SUJETO 1} & \multicolumn{3}{|c|}{ SUJETO 3} & \multicolumn{3}{|c|}{ SUJETO 4} \\
\hline & HR+ & HR- & V. EDA & HR+ & HR- & V. EDA & HR+ & HR- & V. EDA \\
\hline $\begin{array}{c}\text { Incomprensión } \\
\text { de la tarea }\end{array}$ & $x$ & & $x$ & $x$ & & $x$ & $x$ & & $x$ \\
\hline $\begin{array}{c}\text { Motivación } \\
\text { ante la } \\
\text { actividad }\end{array}$ & $x$ & & $x$ & $x$ & & & $x$ & & $x$ \\
\hline $\begin{array}{l}\text { Momentos } \\
\text { de espera de } \\
\text { turno }\end{array}$ & $x$ & & & $x$ & & & & & \\
\hline $\begin{array}{l}\text { Comienzo de } \\
\text { una dinámica } \\
\text { (inicia su com- } \\
\text { pañero) }\end{array}$ & $x$ & & & $x$ & & & & & \\
\hline $\begin{array}{c}\text { Segundos } \\
\text { después de } \\
\text { agredir al } \\
\text { compañero }\end{array}$ & & & & $x$ & & & & & \\
\hline $\begin{array}{l}\text { Momento fi- } \\
\text { nal: juego con } \\
\text { Ipad }\end{array}$ & & & & $x$ & & $x$ & & & \\
\hline
\end{tabular}




\begin{tabular}{|c|c|c|c|c|c|c|c|c|c|}
\hline \multirow{2}{*}{$\begin{array}{c}\text { Situaciones/ } \\
\text { Conductas }\end{array}$} & \multicolumn{3}{|c|}{ SUJETO 1} & \multicolumn{3}{|c|}{ SUJETO 3} & \multicolumn{3}{|c|}{ SUJETO 4} \\
\hline & HR+ & HR- & V. EDA & HR+ & HR- & V. EDA & HR+ & HR- & V. EDA \\
\hline $\begin{array}{c}\text { Comprensión } \\
\text { de la tarea }\end{array}$ & & $x$ & & & & & & $x$ & \\
\hline $\begin{array}{l}\text { Fijación de la } \\
\text { atención en el } \\
\text { Ipad (MAO) } \\
\text { mientras } \\
\text { espera su } \\
\text { turno }\end{array}$ & & $x$ & & & $X$ & & & $X$ & \\
\hline $\begin{array}{c}\text { Transición } \\
\text { entre tareas } \\
\text { (en la mayoría } \\
\text { de ocasiones } \\
\text { centra } \\
\text { atención en } \\
\text { Ipad) }\end{array}$ & & $X$ & & & $x$ & & & & \\
\hline Salto de turno & & & & & & $X$ & & & \\
\hline $\begin{array}{c}\text { Abandono } \\
\text { lugar de } \\
\text { trabajo }\end{array}$ & & & $x$ & & & & & & \\
\hline $\begin{array}{l}\text { Actividad } \\
\text { próxima a su } \\
\text { fin }\end{array}$ & & & $X$ & & & $X$ & & & \\
\hline $\begin{array}{c}\text { Explicación } \\
\text { nueva } \\
\text { dinámica }\end{array}$ & & & & & & & & & $x$ \\
\hline
\end{tabular}

Tal y como se puede observar, todos los sujetos padecen un nivel alto de excitación cuando la tarea no se comprende. A nivel conductual, en general, no se produce ninguna no_ISRC que refleje este estado emocional. Por otro lado, este desajuste también se produce cuando los sujetos esperan su turno. En cambio, cuando la actividad les despierta interés, todos ellos presentan en algún momento conductas no_ISR, en concreto, estereotipias verbales (EV) y motoras (EM). Esta excitación coincide con los parámetros fisiológicos captados en esas situaciones.

En relación a los momentos de calma (HR-), cabe destacar que muchos de ellos se producen cuando los alumnos tienen la mirada atenta al objeto (MAO) (Conducta no_ISR), en este caso en el Ipad. 


\section{Discusión y conclusiones}

No cabe duda de que la labor de las tecnologías en el ámbito de la intervención educativa en niños con TEA es un campo de estudio de creciente interés científico (Sanromà-Giménez et al., 2018). Es por ello, que esta investigación pretende ser un aporte novedoso que permita analizar y comprender la importancia del uso de las tecnologías en personas con TEA (Spiel et al., 2017). Concretamente, se ha pretendido constatar la gran utilidad que ofrece la tecnología, en este caso los wearables, como herramientas para evaluar el impacto de una intervención educativa con niños TEA considerando los parámetros fisiológicos recogidos por el dispositivo Empatica.

Se ha apostado por el uso de wearables, no solo porque facilitan la recogida de datos fisiológicos (Schneider et al., 2015) de los cuales poder obtener estados emocionales, sino porque también permiten realizar mediciones en entornos naturales gracias a su comodidad de uso y portabilidad (Pijeira-Díaz et al., 2017). Este último aspecto debemos considerarlo esencial en las intervenciones con niños TEA, donde el ambiente debe ser muy estructurado y conocido -como es el aula-, ya que los wereables no se convierten en elementos distorsionadores, tal y como indican Poh, Swenson y Picard (2010), en las evaluaciones y mediciones a realizar.

En lo relativo a los datos obtenidos con la Empatica, en el estudio exploratorio que ha vinculado el HR y la EDA a momentos determinados de las sesiones de intervención, es necesario subrayar que nos ha permitido conocer cómo se sienten los sujetos en situaciones concretas, asociando las fluctuaciones de estos parámetros a estados emocionales (Krupa et al., 2016). Por un lado, los niños con TEA han mostrado intranquilidad y estrés (aumento de HR y variación de la EDA) en momentos de incomprensión o dificultad en las tareas y en las esperas y respeto de los turnos, siendo este último una de las problemáticas presentes en personas con TEA (Asociación Americana de Psiquiatría, 2014). Asimismo, y como aspecto a destacar, cuando los sujetos han tenido centrada la atención en el Ipad su estado ha sido más relajado (HR en descenso), coincidiendo con la idea defendida por Cihak et al. (2010), de que el uso de tecnología implica una mejora en conductas como la ansiedad o la angustia.

Igualmente, la asociación de las variaciones de estos parámetros con los estados emocionales permitirá adaptar posteriores intervenciones, al poder conocer cómo se encuentran los niños con TEA en todo momento. Por ello, no podemos obviar la importancia de estudios de este tipo que ayudan a comprender mejor los niveles de excitación emocional, sobre todo en personas con TEA los cuales presentan dificultades a nivel comunicativo.

Por otro lado, esta investigación ha querido constatar el impacto de las tecnologías en un aspecto concreto, el de las hh.ss., altamente afectado en las personas con este trastorno (Rodríguez, 2016). En este sentido, los resultados obtenidos apoyan la concepción del uso de las TIC como instrumento eficaz de mejora en el campo de la comunicación e interacción social (Jiménez et al, 2017). Todos los sujetos han experimentado un aumento significativo en las conductas relacionadas con la competencia social, ya sea con el investigador o con su compañero.

Si profundizamos en los resultados obtenidos, aparece un descenso en la mirada perdida de los sujetos, en favor del mantenimiento de su atención en el recurso tecnoló- 
gico, suscitado por la clara atracción que muestran los niños con TEA hacia los sistemas tecnológicos (Pennisi et al., 2015). El uso de tecnología ha potenciado la disminución en las conductas de abandono de trabajo, como demuestran otras investigaciones (Neely et al, 2013) debido al carácter motivador de las herramientas digitales que utilizan de forma predominante el lenguaje viso-espacial y auditivo (Saladino, 2019) y a su simplicidad (Boyd et al., 2015).

Por otro lado, el gran descenso observado en las estereotipias corrobora la idea de autores como Hetzroni y Tannous (2004) los cuales defienden que el uso de la tecnología disminuye el número de ecolalias. Asimismo, el descenso de conductas o comportamientos inadecuados en varios de los niños se relaciona con otros estudios que describen mejoras conductuales en términos de agresión y negatividad a participar (Cihak et al., 2010), así como un aumento de la tolerancia a las variaciones y una mayor capacidad de inhibición (Suárez et al., 2015) cuando se utilizan recursos digitales.

Otro de los aspectos a destacar es el incremento significativo en la atención conjunta de los sujetos que han participado en la investigación, tanto con el investigador como con su compañero. Múltiples estudios (Passerino et al., 2018) avalan el uso de tecnologías y su relación con el aumento de conductas de atención conjunta en personas con TEA. Del mismo modo, la intervención con herramientas digitales también ha incrementado el contacto ocular, reforzando así la idea defendida por autores como Daniels et al. (2018).

Por otra parte, la triangulación de los datos fisiológicos con las conductas observadas ha permitido advertir tendencias respecto a los estados emocionales de los sujetos en determinadas situaciones, aunque es necesario subrayar que las generalizaciones no son posibles dada la especificidad del trastorno en cada niño. Asimismo, se han observado que, durante gran parte de las sesiones de intervención, los alumnos presentaban un estado de excitación (aumento de HR y variación del EDA), aunque este no se manifestara en conductas observables, demostrando así la importancia del uso de wearables para conocer cómo se sienten los niños con TEA en cada instante.

Finalmente, y en lo que concierne a las limitaciones de la investigación, cabe subrayar que no se ha contado con grupo control, debido, en parte, a la casuística de los sujetos participantes. Como prospectiva de futuro, y dado que son muy escasas las investigaciones que usan los parámetros fisiológicos detallados anteriormente, se considera necesario profundizar en la discriminación de estados emocionales en tiempo real a partir de dichos indicadores que faciliten y mejoren las intervenciones, así como evaluar el impacto concreto de las tecnologías en otros aspectos o ámbitos en los que las personas con TEA presentan dificultades.

Por todo ello, entendemos que es necesario seguir investigando en este ámbito para poder ofrecer a los alumnos con TEA intervenciones educativas acordes a sus necesidades.

\section{Referencias}

Álvarez, C. y San Fabián, J. L. (2012). La elección del estudio de caso en investigación educativa. Gazeta de Antropología, 28(1), 1-12.

Arias-Pujol, E., Fieschi, E., Castelló, C., Miralbell, J., Soldevila, A., Sánchez-Caroz, E., Anguera, M.A. y Mestres, M. (2015). Efectos de la imitación en la interacción social 
recíproca en un niño con Trastorno del Espectro Autista Grave. Revista de Psicopatología y salud mental del niño y del adolescente, (25), 9-20.

Aparicio, M., Dethorne, L.S., Karahalios, K. y Kim, J.G. (2017). Skin Conductance as an In Situ Marker for Emotional Arousal in Children with Neurodevelopmental Communication Impairments: Methodological Considerations and Clinical Implications. ACM Transactions on Accessible Computing, 9(3), 8-29.

Asociación Americana de Psiquiatría (2014). Guía de consulta de los criterios diagnósticos del DSM 5. Arlington, Va: Asociación Americana de Psiquiatría.

Bermúdez, K. B., Aviña, V., Chiquet, R., Olivas, S. y Sánchez, F. (2020). Efecto del "Entrenamiento en Habilidades Conductuales" sobre el establecimiento de habilidades sociales en niños con autismo. Revista Latina de Análisis del Comportamiento, 28(1), 23-36.

Boucsein, W. (2012). Electrodermal activity. Nueva York: Springer.

Boyd, T., Barnett, J. y More, C. (2015). Evaluating iPad technology for enhancing communication skills of children with Autism Spectrum Disorders. Intervention in School and Clinic, 51(1),19-27. https://doi.org/10.1177/1053451215577476

Caballero-González, Y.A. y García-Valcárcel, A. (2020) ¿Aprender con robótica en Educación Primaria? Un medio de estimular el pensamiento computacional. Education in the Knowledge Society, 20, 1-15. https://doi.org/10.14201/eks.22956

Cihak, D., Fahrenkrog, C., Ayres, K. M. y Smith, C. (2010). The use of video modeling via a video iPod and a system of least prompts to improve transitional behaviors for students with autism spectrum disorders in the general education classroom. Journal of Positive Behavior Interventions, 12(2), 103-115. https://doi. org/10.1177/1098300709332346

Cornago, A., Navarro, M. y Collado, F. (2012). Manual de teoría de la mente par niños con autismo: ejercicios, materiales y estrategias. Valencia: Psylicom.

Crescenzi-Lanna, L. y Grané-Oró, M. (2016). Análisis del diseño interactivo de las mejores apps educativas para niños de cero a ocho años. Comunicar, 24(46), 77-85. https://doi.org/10.3916/C46-2016-08

Daniels, J., Schwartz, J. N., Voss, C., Haber, N., Fazel, A., Kline, A. y Wall, D.P. (2018). Exploratory study examining the at-home feasibility of a wearable tool for socialaffective learning in children with autism. NpjDigital Medicine, 1(1), 1-10. https:// doi.org/10.1038/s41746-018-0035-3

De la Iglesia, M. y Olivar, J.S. (2008). Intervención sociocomunitaria en los trastornos del espectro autista de alto funcionamiento. Revista de patología y psicopatología clínica, 13(1), 1-19. https://doi.org/10.5944/rppc.vol.13.num.1.2008.4046

Garbarino, M., Lai, M., Tognetti, S., Picard, R.W. y Bender, D. (2014). Empatica E3 - A wearable Wireless multi-sensor device for real-time computerized biofeedback and data acquisition. En Wireless Mobile Communication and Healthcare (Mobihealth), 2014 EAI 4th International Conference on, (pp. 39-42). Atenas, Grecia: IEEE. https://doi. org/10.4108/icst.mobihealth.2014.257418

Hardy, C., Ogden, J., Newman, J. y Cooper, S. (2002). Autism and ICT: A guide for teachers and parents. Nueva York: David Fulton. 
Hetzroni, O. y Tannous, J. (2004). Effects of a Computer-Based Intervention Program on the Communicative Functions of Children with Autism. Journal of Autism and Developmental Disorders, 34(2), 95-113. https://doi.org/10.1023/b:jadd.0000022602.40506.bf

Jiménez, M. D., Serrano, J. L. y Prendes, M. P. (2017). Estudio de caso de la influencia del aprendizaje electrónico móvil en el desarrollo de la comunicación y el lenguaje con un niño con TEA. Educar, 53(2), 419-443. https://doi.org/10.5565/rev/educar.782

Jodra, M. (2015). Cognición temporal en personas adultas en autismo: Un análisis experimental [Doctoral dissertation, Universidad Complutense de Madrid].

Jodra, M. y García-Villamisar, D. (2019). Impacto de la activación emocional en el reconocimiento de emociones en personas adultas con Trastornos del Espectro del Autismo y Discapacidad Intelectual. Acción Psicológica, 16(2), 103-118. https://doi. org/10.5944/ap.16.2.25566

Kelly, J. A. (2000). Entrenamiento en habilidades sociales. Bilbao: Descée de Brouwer.

Koo, S. H., Gaul, K., Rivera, S., Pan, T. y Fong, D. (2018). Wearable technology design for autism spectrum disorders. Archives of Design Research, 31(1), 37-55. https://doi. org/10.15187/adr.2018.02.31.1.37

Kowallik, A. E. y Schweinberger, S. R. (2019). Sensor-Based Technology for Social Information Processing in Autism: A Review. Sensors, 19(21), 4787. https://doi. org/10.3390/s19214787

Krupa, N., Anantharam, K., Sanker, M., Datta, S. y Vijay, J. (2016). Recognition of emotions in autistic children using physiological signals. Health Technology, 6(2), 137-147. https://doi.org/10.1007/s12553-016-0129-3

Liesa, M. (2002). Potenciación del aprendizaje estratégico y de las habilidades sociales de los niños con necesidades educativas especiales (síndrome de down), en su integración en secundaria [Doctoral dissertation, Universidad de Zaragoza].

López, B. y Leekam, S.R. (2007). Teoría de la coherencia central: una revisión de los supuestos teóricos. Journal for the Study of Education and Development, 30(3), 439-457. https://doi.org/10.1174/021037007781787462

Mendo, S. (2019). Desarrollo de Habilidades Sociales y de Trabajo en Equipo en el contexto universitario: Aprendizaje Cooperativo y Entrenamiento en Habilidades Sociales [Doctoral dissertation, Universidad de Extremadura]. Universidad de Extremadura.

Monjas, M. I. (2009). Como promover la convivencia: Programa de Asertividad y Habilidades Sociales (PAHS). Educación Infantil, Primaria y Secundaria. Madrid: CEPE.

Neale, H. R., Leonard, A. y Kerr, S. (2002). Exploring the role of virtual environments in the special needs classroom. En P. Sharkey, C. Sik Lányi y P. Standen (Eds.), Proceedings of the 4th international conference on disability, virtual reality and associated technologies (pp. 259-266). Reading: The University of Reading.

Neely, L., Rispoli, M., Camargo, S., Davis, H. y Boles, M. (2013). The effect of instructional use of an $\mathrm{iPad} \AA$ on challenging behavior and academic engagement for two students with autism. Research in Autism Spectrum Disorders, 7(4), 509-516. https:// doi.org/10.1016/j.rasd.2012.12.004

Parsons, S., Yuill, N., Good, J., Brosnan, M., Austin, L., Singleton, C. y Bossavit, B. (2016). What Technology for Autism Needs to be Invented? Idea Generation from the Autism Community via the ASCmeI.T. App. En K. Miesenberger y G. Kourou- 
petroglou (Eds.), Computers Helping People with Special Needs, (pp. 343-350). Cham: Springer. https://doi.org/10.1007/978-3-319-41267-2_49

Passerino, L. M., Guimarães Júnior, C. S. S. y Baldassarri, S. (2018). Mesas tangibles para la planificación cognitiva en alumnos con trastorno del espectro autista (TEA). En P. Arnaiz y M. D. Gracia (Coords.). Tecnología accesible e inclusiva: logros, resistencias y desafíos, (pp. 1-12). Murcia: Educarms.

Pedrosa, I., Borges del Rosal, Á., Herranz, N., Lorenzo, M. y García-Cueto, E. (2013). Desarrollo del Protocolo de Observación de Interacción en el Aula: aplicación en un programa de niños con altas capacidades. Revista de Educación (Núm. extraordinario), 293-321.

Pennisi, P., Tonacci, A., Tartarisco, G., Billeci, L., Ruta, L., Gangemi, S. y Pioggia, G. (2015). Autism and social robotics: A systematic review. Autism Research, 9(2), 165183. https://doi.org/10.1002/aur.1527

Peñaherrera-Vélez, M.J., Cobos-Cali, M., Dávila-Pontón, Y. y Vélez- Calvo, X. (2019). Intervención en las habilidades sociales de las altas capacidades. Un estudio de caso. Revista INFAD de Psicología, 5(1), 401-410. https://doi.org/10.17060/ijodaep.2019. n1.v5.1618

Peters, L. y Thompson, R. (2015). Teaching children with autism to respond to conversation partners' interest. Journal of Applied Behavior Analysis, 48(3), 544-562. https:// doi.org/10.1002/jaba.235

Pijeira-Díaz, H.J., Drachsler, H., Kirschner, P.A. y Järvelä, S. (2017). Profiling sympathetic arousal in a physics course: How active are students. Journal of Computer Assisted Learning, 34(4), 397-408. https://doi.org/10.1111/jcal.12271

Poh, M.-Z., Swenson, N. C. y Picard, R. W. (2010). A wearable sensor for unobtrusive, long-term assessment of electrodermal activity. IEEE Transactions on Biomedical Engineering, 57(5), 1243-1252.

Rodríguez, A. (2012). Orientación profesional por competencias transversales para mejorar la empleabilidad [Doctoral dissertation, Universidad de Zaragoza]. Universidad de Zaragoza.

Rodríguez, F. (2016). Guía de intervención logopédica en los Trastornos del Espectro del Autismo. Madrid: Síntesis.

Saladino, M., Marín, D. y San Martín, A. (2019). Aprendizaje mediado por tecnología en alumnado con TEA. Una revisión bibliográfica. Etic@net: Revista científica electrónica de Educación y Comunicación en la Sociedad del Conocimiento, 19(1), 1-25.

Sano, A. y Picard, R.W. (2013). Stress Recognition using Wearable Sensors and Mobile Phones. En IEEE (Coord.). Humaine Association Conference on Affective Computing and Intelligent Interaction, (pp. 671-676). Switzerland: CPS.

Sanromà-Giménez, M., Lázaro-Cantabrana, J. L. y Gisbert-Cervera, M. (2018). El papel de las tecnologías digitales en la intervención educativa de niños con trastorno del espectro autista. Revista Interuniversitaria De Investigación En Tecnología Educativa, (4), 41-54. https://doi.org/10.6018/riite/2018/327991

Sarabadani, S., Schudlo, L. C., Samadani, A. A. y Kushski, A. (2018). Physiological Detection of Affective States in Children with Autism Spectrum Disorder. IEEE Transactions on Affective Computing, 1-14. https://doi.org/10.1109/taffc.2018.2820049 
Schneider, J., Börner, D., Van Rosmalen, P. y Specht, M. (2015) Augmenting the senses: A review on sensor-based learning support. Sensors, 15, 4097-4133. https://doi. org/10.3390/s150204097

Soto, A., Camerino, O., Iglesias, X., Anguera, M.T. y Castañer, M. (2019). LINCE PLUS: Research Software for Behavior Video Analysis. Apunts. Educación Física y Deportes, 137, 149-153. https://doi.org/10.5672/apunts.2014-0983.es.(2019/3).137.11

Spiel, K., Frauenberger, C. y Fitzpatrick, G. (2017). Experiences of autistic children with technologies. International Journal of Child-Computer Interaction, 11, 50-61. https://doi. org/10.1016/j.ijcci.2016.10.007

Suárez, F., Mata, B. y Peralbo, M. (2015). Valoración de un programa de intervención para niños con TEA basado en las TIC. Revista de Estudios e Investigación en Psicología y Educación, (9), 094-098. https://doi.org/10.17979/reipe.2015.0.09.650

Uribe, D.S., Gómez, M. y Arango, O.E. (2010). Teoría de la Mente: Una revisión acerca del desarrollo del concepto. Revista Colombiana de Ciencias Sociales, 1(1), 28-37.

Vahabzadeh, A., Keshav, N. U., Abdus-Sabur, R., Huey, K., Liu, R. y Sahin, N.T. (2018). Improved Socio-Emotional and Behavioral Functioning in Students with Autism Following School-Based Smartglasses Intervention: Multi-Stage Feasibility and Controlled Efficacy Study. Behavioral Sciences, 8(85), 1-17. https://doi.org/10.3390/ bs8100085

Yin, R.K. (2008). Case Study Research: Design and methods. Thousand Oaks: Sage.

Fecha de recepción: 5 de junio de 2020.

Fecha de revisión: 4 de julio de 2020.

Fecha de aceptación: 21 de octubre de 2020. 